\title{
A influência da morfina ou tramadol pela via epidural no trânsito gastrintestinal de equinos
}

\author{
[Influence of epidural morphine or tramadol in gastrointestinal transit of horses] \\ C.M. Bertonha', C.A.A. Valadão ${ }^{2}$, M.H. Cruz ${ }^{3}$, M. Takeda ${ }^{3}$, B.F. Cholfe ${ }^{3}$ \\ ${ }^{1}$ Aluna de pós-graduação - UNESP Câmpus de Jaboticabal, SP \\ ${ }^{2}$ UNESP - Câmpus de Jaboticabal, SP \\ ${ }^{3}$ Médico veterinário autônomo - São José do Rio Preto, SP
}

\begin{abstract}
RESUMO
Os opioides são utilizados na medicina veterinária na analgesia dos animais, porém há restrição quanto ao uso desses fármacos em equinos, pois podem desencadear alterações gastrintestinais, mas a administração pela via epidural minimiza os efeitos adversos. Assim, oito equinos hígidos foram utilizados em três grupos experimentais, objetivando-se avaliar a influência no trânsito gastrintestinal da injeção epidural de morfina $(0,2 \mathrm{mg} / \mathrm{kg})$, tramadol $(1,0 \mathrm{mg} / \mathrm{kg})$ ou $\mathrm{NaCl} 0,9 \%$. Para a avaliação do trânsito gastrintestinal, foi administrada a lignina purificada e enriquecida (Lipe $\left.{ }^{\circledR}\right)$, pela via oral, a qual posteriormente foi analisada nas fezes. As fezes foram coletadas para investigação desse marcador, antes da epidural e em intervalos até totalizar 48 horas do início do experimento. Os dados foram submetidos à análise de variância (ANOVA) de uma via, com repetições múltiplas. As médias entre cada momento dos grupos e dos momentos dentro de cada grupo foram comparadas pelo teste de Student-Newman-Keuls $(\mathrm{P} \leq 0,05)$. A morfina ou o tramadol administrados pela via epidural não alteraram o tempo médio de retenção da fase líquida, a taxa de passagem e o tempo de trânsito do marcador utilizado. Diante de tais achados, concluise que a morfina ou o tramadol pela via epidural não alteram o trânsito gastrintestinal.
\end{abstract}

Palavras-chave: opioide, espinhal, cavalo

\begin{abstract}
Opioids provide analgesia and are used in veterinary, however there is a restriction regarding the use of these drugs in horses, because may lead to ileus, but epidural opioids minimize those side effects. Eight healthy horses were used in three experimental groups, to evaluate the gastrointestinal influence after epidural morphine $(0.2 \mathrm{mg} / \mathrm{kg})$, tramadol $(1.0 \mathrm{mg} / \mathrm{kg}$ ) or $\mathrm{NaCl} 0.9 \%$. Intestinal transit was evaluated with the administration of Lipe ${ }^{\circledR}$ (purified enriched isolated lignin) and later analyzed in the feces. Feces were collected before the epidural and at intervals up to 48 hours after the beginning of the experiment. The data were analyzed by a one-way analysis of variance or Friedman or Kruskal-Wallis and StudentNewman Keuls test as a post hoc. A value of $P \leq 0.05$ was considered significant. Epidural morphine or tramadol did not change the mean time for retention of the liquid phase, passage rate, and the transit time of the marker used. In conclusion, epidural morphine or tramadol does not change gastrointestinal transit.
\end{abstract}

Keywords: opioid, spinal, gastrointestinal, equine

\section{INTRODUÇÃO}

A dor é considerada uma experiência subjetiva, com características individuais (Sousa, 2002). Quando não tratada, ela pode ter consequências fisiológicas negativas em equinos, como o atraso

Recebido em 19 de julho de 2017

Aceito em 26 de outubro de 2017

E-mail: candice-vet@hotmail.com na recuperação cirúrgica ou efeitos deletérios em longo prazo. E como os sinais clínicos da dor nos equinos podem ser sutis ou imperceptíveis, ocorre frequentemente negligência do uso de analgésicos nessa espécie. Com o uso de analgésicos, há benefícios, que incluem manutenção do escore corporal, redução do 
período de internação e do custo do tratamento, além de respeito a questões éticas e humanitárias (Taylor, 2005; Matthews e Carroll, 2007).

$\mathrm{O}$ bloqueio espinhal produz anestesia e analgesia, e seu uso na medicina veterinária tem avançado consideravelmente nos últimos anos. Há comprovação da eficácia e segurança dos bloqueios epidurais em equinos, que são adotados pela facilidade de execução, pelo custo baixo e pela praticidade (Natalini, 2010). A anestesia epidural foi descrita e adotada pela primeira vez em equinos no ano de 1925, sendo subsequentemente indicada para manobras obstétricas e procedimentos cirúrgicos da região perineal (Muir, 2011).

Os opioides são amplamente empregados na analgesia epidural, sendo a morfina uma representante dessa classe de fármaco. A morfina é classificada como agonista $\mu$ puro e analgésico potente e foi o primeiro opioide administrado pela via epidural em equinos, como descrito por Valverde et al. (1990). Existe a preocupação constante quanto ao uso da morfina nos equinos, pois ela pode diminuir a motilidade gastrintestinal quando administrada pela via intravenosa (Boscan et al., 2006; Matthews e Carroll, 2007; Matthews, 2009). No entanto, foi descrito que o uso da morfina por via epidural causa apenas redução temporária da motilidade gastrintestinal, sem acarretar atonia de íleo (Sano et al., 2011).

O tramadol é classificado como agonista $\mu$ não opioide e utilizado para o tratamento de dor discreta a moderada em equinos (Matthews, 2009). Esse fármaco interfere minimamente ou de modo insignificante na motilidade gastrintestinal, reduzindo discretamente os borborigmos intestinais quando administrado pela via intravenosa em equinos (Shilo et al., 2007). Entretanto, não há relatos do desenvolvimento do quadro de cólica após a administração de tramadol pela via epidural (Natalini; Robinson, 2000; Giorgi et al., 2010).

A auscultação intestinal é utilizada para avaliar a motilidade gastrintestinal em equinos, porém é um método subjetivo (Sano et al., 2011). Existem métodos objetivos que avaliam o trânsito intestinal, como o uso de marcadores que avaliam qualitativamente e quantitativamente diversos fenômenos biológicos ou nutricionais, e tornam possível a monitoração da taxa de passagem, a fim de identificar e quantificar a concentração nas fezes (Machado et al., 2011).

Há um marcador de fase líquida denominado de lignina purificada e enriquecida (Lipe $\AA)$, que se mostrou eficiente indicador de digestibilidade em equinos. Ele substitui o método de coleta total das fezes, tornando possível o cálculo da produção fecal pela espectroscopia no infravermelho. $\mathrm{O}$ método Lipe ${ }^{\circledR}$ possui baixo custo, além de necessitar de curto período de adaptação dos animais. $\mathrm{Na}$ validação desse método para equinos, a recuperação fecal do marcador foi semelhante ao obtido com a coleta de fezes. A taxa de recuperação fecal do Lipe ${ }^{\circledR}$ foi de $95,94 \%$, enquanto a taxa de recuperação fecal do óxido crômico foi de 83,73\%. (Lima et al., 2008; Lanzetta et al., 2009; Machado et al., 2011).

Considerando que os opioides podem reduzir a motilidade intestinal em equinos e que há poucas informações na literatura, este estudo buscou avaliar as alterações no trânsito gastrintestinal resultantes da administração de tramadol ou de morfina em equinos pela via epidural.

\section{MATERIAL E MÉTODOS}

Este estudo foi aprovado pela Comissão de Ética no Uso de Animais (Ceua) da Faculdade de Ciências Agrárias e Veterinárias (FCAV) da Universidade Estadual Paulista "Júlio de Mesquita Filho" - Câmpus de Jaboticabal, número 008401/13.

Foram utilizados oito equinos adultos (dois machos castrados e seis fêmeas não prenhes), com peso médio de $336,3 \pm 33,4 \mathrm{~kg}$ e idade de $5,5 \pm 1,3$ anos. Os equinos apresentavam boa condição corporal e permaneciam em piquetes, onde recebiam feno (Cynodon sp.), sal mineral para equinos (Supra Sal - Supra, Brasil) e água ad libitum.

Os animais foram usados em três grupos experimentais: grupo tramadol (GT), grupo morfina (GM) ou no grupo controle (GC), com intervalo de, no mínimo, 15 dias entre cada ensaio experimental, que foi realizado no período matutino para evitar variações circadianas. $\mathrm{Na}$ tarde anterior, o cateter foi implantado, após tricotomia da região sacrococcígea e antissepsia 
com clorexidine degermante e clorexidine alcoólico a $0,5 \%$. O espaço sacrococcígeo foi identificado após movimentação da cauda na direção dorsoventral, para, então, realizar-se infitração $3 \mathrm{~mL}$ de cloridrato de lidocaína a $2 \%$ (Xylestesin 2\% - Cristália Produtos Químicos e Farmacêuticos Ltda. - Brasil) no espaço subcutâneo das vértebras sacrococcígeas. Após cinco minutos da anestesia da área, perfurou-se a pele com bisturi, para a colocação da agulha espinhal de Tuohy (Agulha Peridural BD ${ }^{\mathrm{TM}}$ Tuohy 16G - BD, Brasil) no espaço epidural. Logo depois da confirmação de sua correta localização, que foi pela ausência de resistência e teste da gota pendente, introduziu-se o cateter epidural (Cateter epidural Perifix 18G - B Braun Sharing Expertise, Alemanha) cranialmente (15 centímetros) e realizou-se a fixação dele à pele. Até o momento do início do experimento, os animais foram mantidos em piquetes com livre acesso à água e à alimentação.

Não foi realizado jejum nos animais, que receberam a mesma dieta até o momento em que foram colocados no tronco de contenção, em condições naturais de climatização. Inicialmente, realizou-se sondagem nasogástrica, para administração de cinco gramas de Lipe ${ }^{\circledR}$ (Produtos de Pesquisas Simões Saliba Florestal, MG, Brasil), diluídos em um litro de água. A sonda foi retirada ao término da administração. Imediatamente após a administração do marcador de trânsito intestinal, administrou-se 0,2 mg/kg de morfina (Dimorf Cristália Produtos Químicos e Farmacêuticos Ltda., Brasil) no GM ou 1,0mg/kg de tramadol no GT (Tramadon - Cristália Produtos Químicos e Farmacêuticos Ltda. - Itapira, SP, Brasil) ou $\mathrm{NaCl}$ 0,9\% (Solução Fisiológica, Cloreto de Sódio 0,9\% - Laboratório Sonobiol Ltda. Pouso Alegre, MG, Brasil) no GC pela via epidural.

A aplicação foi padronizada em dois minutos e o volume final (mL) foi ajustado com $\mathrm{NaCl} 0,9 \%$, de acordo com a equação $3,4+(0,013 \times$ peso do animal em kg) (Gomez de Segura et al., 1998). Em seguida, administrou-se solução de $\mathrm{NaCl}$ $0,9 \%$, calculada de acordo com o tamanho do cateter (aproximadamente $1 \mathrm{~mL}$ ), com o objetivo de empurrar o volume residual do tratamento do interior do cateter. Nos primeiros 60 minutos, os animais foram mantidos no tronco de contenção e, logo após, retornaram ao piquete, onde receberam feno (Cynodon sp.), sal mineral para equinos e água ad libitum.

Para avaliação do trânsito intestinal, foram coletadas fezes em MB (antes da administração do Lipe ${ }^{\circledR}$ pela via oral), 30, 60, 120, 240, 360, 480, 720, 960, 1440 e 2880 minutos após a injeção epidural, pela palpação transretal, diretamente do reto do animal, as quais foram condicionadas em tubo coletor (Frasco Coletor $80 \mathrm{~mL}$ - Prolab, Brasil). As fezes foram congeladas $\left(-20^{\circ} \mathrm{C}\right)$ por três meses para futuro processamento. Após o descongelamento das fezes em temperatura ambiente, as amostras foram pré-secas em estufa de ventilação forçada a $55^{\circ} \mathrm{C}$, moídas em malha de 1-mm e enviadas ao laboratório para análise, onde o examinador desconhecia os tratamentos realizados. Adicionou-se brometo de potássio a $1 \%$ nas fezes, que foram submetidas à espectroscopia no infravermelho. A razão logarítmica das bandas espectrais entre os comprimentos de onda $\lambda 1(1050$ nm) e $\lambda 3(1650$ nm) foi utilizada para a determinação da concentração do marcador. $\mathrm{O}$ tempo médio de retenção (TMR) da fase líquida das amostras foi calculado, utilizando-se a equação: TMR (horas) $=\Sigma \mathrm{mi} \times \mathrm{ti} / \Sigma \mathrm{mi}$, em que: mi=quantidade de indicador excretado no intervalo de tempo e ti=intervalo de tempo entre o fornecimento do indicador e o tempo de coleta fecal. A taxa de passagem (TxP) da fase líquida foi estimada com o inverso da TMR: TxP $(\% \mathrm{~h}-$ $1)=1 / \mathrm{TMR}$. O tempo de trânsito (TT) foi estimado como o intervalo entre o fornecimento do indicador e o aparecimento inicial nas fezes (Saliba et al., 2003; Godoi et al., 2009).

Utilizou-se o Scientific Data Analysis and Graphing Software (Sigma Plot 11.0) para a realização das análises estatísticas. Os dados foram submetidos inicialmente ao teste de normalidade Shapiro-Wilk. O trânsito intestinal foi submetido à análise de variância (ANOVA) de uma via, com repetições múltiplas. As médias entre cada momento dos grupos e dos momentos dentro de cada grupo foram comparadas pelo teste de Student-Newman-Keuls $(\mathrm{P} \leq 0,05)$, para detecção das diferenças significativas.

\section{RESULTADOS E DISCUSSÃO}

De fato, o uso de opioides pode reduzir a motilidade intestinal, pois há receptores opioides no sistema nervoso entérico, os quais causam 
redução da contratilidade da musculatura lisa e modificam a atividade propulsiva intestinal (Wood e Galligan, 2004). Em diversos segmentos intestinais, há neurônios que possuem receptores nicotínicos colinérgicos e a inibição desses neurônios ocasionada pela morfina resulta na diminuição do tônus colinérgico, por reduzir a liberação da acetilcolina (Calignano et al., 1991).

Porém, com a administração epidural ocorre facilitação da ligação do fármaco, devido à proximidade dos receptores sensoriais localizados no neuroeixo. Assim, as doses empregadas são significativamente menores, bem como os efeitos adversos, o que reduz o risco de complicações para o paciente equino (Robinson e Natalini, 2002).

Diversos estudos anteriores, que empregaram a injeção epidural de morfina, nas diferentes espécies, incluindo cães e equídeos, estabeleceram a dose de $0,1 \mathrm{mg} / \mathrm{kg}$ como eficaz para a produção de analgesia (Natalini e Robinson, 2000; Valadão et al., 2002; Guirro et al., 2011). Entretanto, Sano et al. (2011) utilizaram a dose de $0,2 \mathrm{mg} / \mathrm{kg}$ de morfina pela via epidural em equinos para avaliar, de forma inédita, os efeitos do fármaco no trânsito gastrintestinal, e relataram atraso inicial da motilidade intestinal. Assim, buscou-se utilizar diferente metodologia para avaliar os efeitos no trânsito gastrintestinal em equinos após a injeção epidural de $0,2 \mathrm{mg} / \mathrm{kg}$ de morfina com o uso do marcador Lipe $\AA$.
A produção de analgesia é comprovada após o uso do tramadol pela via epidural na dose de $1,0 \mathrm{mg} / \mathrm{kg}$, em equinos (Natalini e Robinson, 2000), mas não há estudos objetivos na avaliação do trânsito gastrintestinal após o uso desse fármaco. Devido à escassez de informações sobre os possíveis efeitos da injeção epidural do tramadol $(1,0 \mathrm{mg} / \mathrm{kg})$ sobre o trânsito intestinal de equinos, buscou-se comparar os efeitos do tramadol com os da morfina $(0,2 \mathrm{mg} / \mathrm{kg})$ injetada pela mesma via nesse parâmetro.

O cálculo de volume final, conforme proposto por Gomez de Segura et al. (1998), foi utilizado para assegurar o espraiamento rostral do opioide no canal vertebral e evitar interferências no resultado. Baseando-se nessa padronização, o volume administrado no espaço epidural não ultrapassou $10 \mathrm{~mL}$. Estudo com maior volume foi realizado por Natalini e Robinson (2000), que utilizaram volume final de $20 \mathrm{~mL}$ na injeção epidural de opioides em equinos e concluíram que esse volume é doloroso, pela compressão dos nervos espinhais sacral e lombar, o que pode influenciar resultados.

As fezes foram processadas e encaminhadas ao Laboratório de Produtos e Pesquisa Simões Saliba, onde foram mensuradas as concentrações de Lipe®. Não foram constatadas diferenças significativas no TMR, TxP e TT entre os tratamentos (Tab. 1).

Tabela 1. Médias do tempo médio de retenção (TMR), taxa de passagem (TxP) e do tempo de trânsito (TT) da fase líquida da digesta, de equinos tratados com injeção epidural de $\mathrm{NaCl} 0,9 \%$ (GC n=8) ou $0,2 \mathrm{mg} / \mathrm{kg}$ de morfina (GM n=8) ou 1,0mg/kg de tramadol (GT n=7), FCAV/Unesp - Jaboticabal, 2014

\begin{tabular}{cccc}
\hline & GC & GM & GT \\
\hline TMR (horas) & 10,49 & 10,81 & 12,07 \\
TxP (\% horas) & 0,096 & 0,096 & 0,083 \\
TT (horas) & 1,5 & 1,5 & 1,5 \\
\hline
\end{tabular}

A avaliação dos movimentos intestinais pela auscultação é um método subjetivo. Diante desse fato, buscou-se complementar a avaliação do trânsito intestinal objetivamente. $\mathrm{O}$ método Lipe® foi eleito para avaliação do trânsito gastrintestinal, pois foi validado anteriormente em equinos (Lanzetta et al., 2009). Com a utilização deste método, foi possível constatar que a taxa de passagem do marcador foi a mesma nos grupos controle, morfina e tramadol, não havendo alterações significativas no trânsito intestinal.

A motilidade intestinal foi avaliada pelo método de auscultação abdominal após a administração do tramadol, pela via intravenosa, na dose de $2,0 \mathrm{mg} / \mathrm{kg}$, e constatou-se diminuição dos escores de movimentos intestinais nos primeiros 60 
minutos (Franco et al., 2014). No presente estudo, com dose inferior e via de administração diferente, não foi observada alteração significativa na avaliação objetiva do trânsito intestinal.

Sano et al. (2011) avaliaram o trânsito intestinal após a administração de morfina pela via epidural $(0,2 \mathrm{mg} / \mathrm{kg})$, por meio da utilização de esferas radiopacas administradas pela via oral e recuperadas nas fezes. O tempo de trânsito das esferas aumentou em cinco horas após a injeção epidural de morfina com relação ao grupo controle e ocorreu atraso no primeiro aparecimento das esferas nas fezes em três horas, mesmo sem alterações na auscultação da motilidade intestinal.

O uso de esferas radiopacas possui algumas limitações, pois a contagem é realizada após a realização de radiografias, podendo ocorrer sobreposição delas, o que prejudica a precisão do resultado. A precisão do atraso do aparecimento das esferas nas fezes após a injeção epidural de morfina em equinos, constatado por Sano et al. (2011), pode ser comprometida pela demora na primeira contagem de esferas, que ocorreu após 12 horas da injeção epidural. No entanto, esse atraso não pôde ser comprovado quando o trânsito gastrintestinal foi medido pelo método Lipe ${ }^{\circledR}$ no presente estudo.

Diferentemente do presente estudo, que não usou sedativos, Sano et al. (2011) utilizaram o agonista de receptores adrenérgicos tipo $\alpha 2$ xilazina $(0,2 \mathrm{mg} / \mathrm{kg})$ pela via intravenosa, para facilitar a injeção epidural, o que pode ter contribuído para o atraso da recuperação das esferas nas fezes. Além disso, vale ressaltar que se trata de duas metodologias distintas, sendo o Lipe $®$ um marcador de fase líquida.

As esferas radiopacas também foram empregadas por Martin-Flores et al. (2014) para avaliar o trânsito intestinal, em equinos, após a injeção epidural de morfina $(0,1 \mathrm{mg} / \mathrm{kg})$ submetidos à criptorquidectomia, via laparoscopia, sob anestesia geral. Com este modelo experimental, não foram constatadas alterações no trânsito intestinal, diferindo dos resultados de Sano et al. (2011), com a mesma metodologia para avaliação desse parâmetro. A diferença dos dois estudos pode ser atribuída a doses diferentes da morfina, à anestesia geral e ao estímulo doloroso presentes no estudo de Martin-Flores et al. (2014).

Uma limitação na determinação do trânsito intestinal é a amostragem da digesta, pois ocorre divisão nas fases líquida e sólida, por isso uma amostragem não representativa da digesta pode acontecer quando se usa um único indicador, sendo o Lipe ${ }^{\circledR}$ um marcador exclusivo de fase líquida. Para minimizar a possibilidade da digesta em se separar durante a coleta, recomenda-se que sejam utilizados indicadores com afinidade pelas diferentes fases da digesta (líquida e sólida) ou, então, que os indicadores estejam distribuídos uniformemente entre as fases (Faichney, 1980). Porém, constatou-se que o marcador de fase líquida Lipe $\AA$ apresentou recuperação nas fezes igual a 95\%, 94\% em equinos (Lanzetta et al., 2009).

\section{CONCLUSÃO}

Não há alteração do trânsito gastrintestinal após a administração de morfina $(0,2 \mathrm{mg} / \mathrm{kg})$ ou tramadol $(1,0 \mathrm{mg} / \mathrm{kg})$, pela via epidural, em equinos saudáveis, por meio do emprego do Lipe ${ }^{\circledR}$ como método objetivo de avaliação. Esse marcador de fase líquida se mostrou seguro e de fácil aplicação e permitiu assegurar que os opioides em questão utilizados pela via epidural não prejudicam o trânsito gastrintestinal. Estudos futuros devem ser realizados para a utilização de novos métodos de avaliação do trânsito gastrintestinal após a administração injeção epidural de morfina ou tramadol, já que tais fármacos produzem analgesia, como demonstrado em estudos prévios.

\section{REFERÊNCIAS}

BOSCAN, P.; VAN HOOGMOED, L.M.; FARVER, TB.; SNYDER, J.R. Evaluation of the effects of the opioid agonist morphine on gastrointestinal tract function in horses. Am. J. Vet. Res., v.67, p.992-997, 2006.

CALIGNANO, A.; MONCADA, S.; DI ROSA, M. Endogenous nitrk: oxide modulates morphine-induced constipation. Biochem. Biophys. Res. Commun., v.181, p.889-893, 1991.

FAICHNEY, G.J. The use of markers to measure digesta flow from the stomach of sheep fed once daily. J. Agric. Sci., v.94, p.313-318, 1980. 
FRANCO, L.G.; MORENO, J.C.D.; TEIXEIRA NETO, A.R. et al. Tramadol effects on clinical variables and the mechanical nociceptive threshold in horse. Ciênc. Rural, v.44, p.517-523, 2014.

GIORGI, M.; SACCOMANNI, G.; ANDREONI, V. Pharmacokinetics of tramadol after epidural administration in horses. J. Equine Vet. Sci., v.30, 2010

GODOI, F.N.; ALMEIDA, F.Q.; SALIBA, E.O. et al. Consumo, cinética digestiva e digestibilidade de nutrientes em equinos atletas alimentados com dieta contendo óleo de soja. Rev. Bras. Zootec., v.10, p.1928-1937, 2009.

GOMEZ DE SEGURA, I.A.; DE ROSSI, R.; SANTOS, M. et al. Epidural injection of ketamine for perineal analgesia in the horse. Vet. Surg., v.27, p.384391, 1998.

GUIRRO, E.C.B.P.; FERREIRA, I.M.M.; VALADÃO, C.A.A. et al. Efeitos comportamental, clínico e analgésico promovidos pela injeção epidural preventiva de morfina, xilazina ou clonidina, em equinos. Ciênc. Rural, v.41, 2011.

LANZETTA, V.A.S.; REZENDE, A.S.C.; SALIBA, E.O.S. et al. Validação do Lipe ${ }^{\circledR}$ como método para determinar a digestibilidade dos nutrientes em equinos. Rev. Bras. Zootec., v.38, p.69-74, 2009.

LIMA, J.B.M.P.; GRAÇA, D.S.; BORGES, A.L.C.C. et al. Uso do óxido crômico e do LIPE® na estimativa do consumo de matéria seca por bezerros de corte. Arq. Bras. Med. Vet. Zootec., v.60, p.1197-1204, 2008 .

MACHADO, A.S.; GODOY, M.M.; LIMA, M.L. et al. Utilização de óxido crómico e LIPE® como indicadores externos na estimativa de digestibilidade em ruminantes. PUBVET, v.5, ed.167, art.1124, 2011.

MARTIN-FLORES, M.; CAMPOY, L.; KINSLEY, M.A. et al. Analgesic and gastrointestinal effects of epidural morphine in horses after laparoscopic cryptorchidectomy under general anesthesia. Vet. Anaesth. Analg., v.41, p.430-437, 2014.

MATTHEWS, N.S. Equine analgesics: what do we have and what do we need? In: ANNUAL CONVENTION OF THE AMERICAN ASSOCIATION OF EQUINE PRACTITIONERS, 53., 2009. Proceedings... Las Vegas: AAEP, 2009.

MATTHEWS, N.S.; CARROLL, G.L. Review of Equine analgesics and pain management. In: ANNUAL CONVENTION OF THE AMERICAN OF THER ASSOCIATION OF EQUINE PRACTITIONERS., 53., 2007, Orlando. Proceeding... Orlando: AAEP, 2007.
MUIR, W.W. Equine local anesthesia and pain management. In: CONGRESS OF THE ASSOCIATION OF EQUINE VETERINARIANS, 17., 2011, Montesilvano. Proceedings.. Montesilvano: [AEV], 2011.

NATALINI, C.C.; ROBINSON, E.P. Evaluation of the analgesic effects of epidurally administered morphine, alfentanil, butorphanol, tramadol and $\mathrm{U} 50488 \mathrm{H}$ in horses. Am. J. Vet. Res., v.61, p.15791586, 2000.

NATALINI, C. C. Spinal anesthetics and analgesics in the horse. Vet. Clin. Equine, v.26, p.551-564, 2010.

ROBINSON, E.; NATALINI, C.C. Epidural anesthesia and analgesia in horses. Vet. Clin. Equine, v.18, p.61-82, 2002.

SALIBA, E.O.S.; PEREIRA, R.A.N.; FERREIRA, W.M. et al. Lignin from Eucaliptus grandis as indicator for rabbits in digestibility trials. Trop. Subtrop. Agroecosyt., v.3, p.107-109, 2003.

SANO, H.; MARTIN-FLORES, M.; SANTOS, L.C.P. et al. Effects of epidural morphine on gastrointestinal transit in unmedicated horses. Vet. Anaest. Analg., v.38, p.121-126, 2011.

SHILO, Y.; BRITZI, M.; EYTAN, B. et al. Pharmacokinetics of tramadol in horses after intravenous, intramuscular and oral administration. $J$. Vet. Pharmacol. Therap., v.31, p.60-65, 2007.

SOUSA, F.A.E.F. Dor: o quinto sinal vital. Rev. Latinoam. Enferm., v.10, p.446-447, 2002.

TAYLOR, P.M. Pharmacological Approaches to Pain Management in the Horse. In: ANNUAL CONVENTION OF THE AMERICAN ASSOCIATION OF EQUINE PRACTITIONERS, 51., 2005, Seattle. Proceedings... Seattle, WA: AAEP, 2005.

VALADÃO, C.A.A.; MAZZEI, S.; OLESKOVICZ, N. Injeção epidural de morfina ou cetamina em cäes: avaliaçäo do efeito analgésico pelo emprego de filamentos de von Frey. Arq. Bras. Med. Vet. Zootec., v.54, p.383-389, 2002.

VALVERDE, A.; LITTLE, C.B.; DYSON, D.H.; MOTTER, C.H. Use of epidural morphine to relieve pain in a horse. Can. Vet. J., v.31, p.2011-212, 1990.

WOOD, J.D.; GALLIGAN, J.J. Function of opioides in the enteric nervous system. Neurogastroenterol Motil, v.16, Supl.2, p.17-28, 2004 